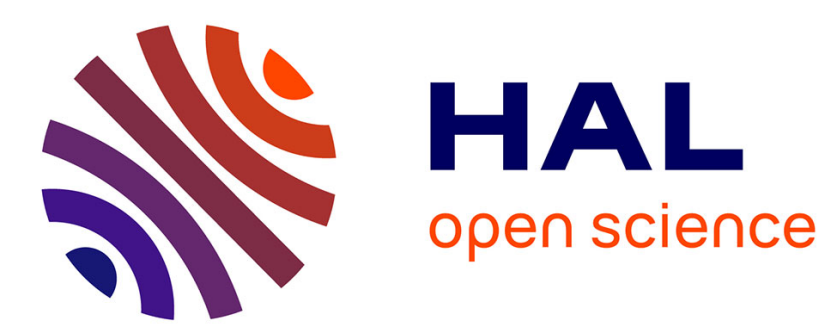

\title{
Assessment of an electromagnetometric system for the investigation of articulatory movements in speech production
}

\author{
Noël Nguyen, Alain Marchal
}

\section{- To cite this version:}

Noël Nguyen, Alain Marchal. Assessment of an electromagnetometric system for the investigation of articulatory movements in speech production. Journal of the Acoustical Society of America, 1993, 94, pp.1152-1152. 10.1121/1.406964 . hal-01392888

\section{HAL Id: hal-01392888 \\ https://hal.science/hal-01392888}

Submitted on 4 Nov 2016

HAL is a multi-disciplinary open access archive for the deposit and dissemination of scientific research documents, whether they are published or not. The documents may come from teaching and research institutions in France or abroad, or from public or private research centers.
L'archive ouverte pluridisciplinaire HAL, est destinée au dépôt et à la diffusion de documents scientifiques de niveau recherche, publiés ou non, émanant des établissements d'enseignement et de recherche français ou étrangers, des laboratoires publics ou privés. 


\title{
Assessment of an electromagnetometric system for the investigation of articulatory movements in speech production
}

\author{
Noël Nguyen ${ }^{\text {a) }}$ and Alain Marchal \\ Laboratoire Parole et Langage, Université de Provence, 29 av. R. Schuman, 13621 Aix-en-Provence, \\ France
}

(Received 22 July 1992; accepted for publication 30 April 1993)

\begin{abstract}
Electromagnetometry can be used to track articulatory movements during speech production by means of small electromagnetic receivers attached to the articulators. This study was aimed at determining the measurement accuracy of the Movetrack system marketed by Botronic (Sweden). The average difference between a position transduced by Movetrack and the actual position, obtained from a precision mechanical device, was less than $0.4 \mathrm{~mm}$, when the receiver was placed in the midsagittal plane and parallel to the transmitters at a distance of about $25 \mathrm{~cm}$ from each transmitter. Higher deviations were observed when the receiver was tilted with respect to the transmitters. Thus the device appears to be capable of delivering accurate measures of position for rigid anatomical structures like the jaw, which are not likely to produce tilt.
\end{abstract}

PACS numbers: 43.70.Jt, 43.70.Aj

\section{INTRODUCTION}

Electromagnetometry, hereafter abbreviated EMA, was introduced recently into phonetics to study articulatory movements in speech production (Hixon, 1971; van der Giet, 1977; Branderud, 1985; Perkell and Cohen, 1986; Perkell et al., 1992; Schönle et al., 1987; Tuller et al., 1990; Hoole, 1993). The articulatory movements are tracked by means of signals induced by alternating electromagnetic fields in small receivers attached to the articulators. The amplitude of each signal is approximately inversely proportional to the cube of the distance between the corresponding receiver and the transmitter. Using two transmitters áctivated by sinusoidal signals of different frequencies (ranging from 10-200 kHz), it is possible to calculate the $x$ and $y$ coordinates of the receivers. The transmitters are placed in the midsagittal plane on a helmet worn by the subject. A complete description of the EMA technique can be found in Perkell et al. (1992).

One of the main difficulties in EMA is the positioning of the receivers on the subject. They must be placed in the midsagittal plane, with their longest axis parallel to the transmitter axes, to obtain the most precise measurements. Violation of any of these requirements may lead to a decrease in the system's accuracy. Thus an off-midline placement of a receiver may give rise to potentially large measurement errors when that receiver is far removed from the midsagittal plane. Substantial errors can also be caused by rotations of a receiver either perpendicular to the plane of attachment (tilts) or parallel to that same plane (twists). While it seems possible to minimize the distance from a receiver to the midline plane, or the degree of twist through proper placement of that receiver, tilting movements ap-

\footnotetext{
a) Now at the following address: Laboratoire de Psycholinguistique, Faculté de Psychologie, Université de Genève, 9 route de Drize, CH-1227 Genève, Switzerland.
}

pear to be much more difficult to avoid. This is particularly true in the oral cavity where deformations of the tongue (Stone, 1991) may put the receivers and transmitters in a nonparallel state. The actual distance between a receiver and each transmitter is then multiplied by $(\cos \theta)^{-1 / p}$ (where $\theta$ is the misalignment angle and $p$ is approximately equal to 3 ), resulting in an error. Two solutions to this problem have been proposed. One consists of using biaxial receivers (Perkell \& Cohen, 1986). The other is to use a third transmitter, placed in the midsagittal plane like the other two, so that tilt effects can be corrected by solving a system of nonlinear equations (Schönle et al., 1987; Perkell et al., 1992).

There are three main electromagnetic systems designed to track articulatory movements at the present time: the system developed by Perkell and his colleagues (not commercially available yet), the Articulograph AG 100, marked by Carstens Medizinelektronik (Germany), and the Movetrack marketed by Botronic (Sweden). The present study was aimed at determining the level of precision of the measurements made by that third system, the Movetrack, which unlike the other two has not been thoroughly evaluated. It is a relatively simple system which does not compensate for tilt effects. It uses two transmitters, one above the head (transmitter Y), and the other behind the head (transmitter $X$ ), each at the end of a U-shaped Plexiglas frame. These frames are attached to a lightweight headset, allowing for head movements. As many as eight receivers can be used. Each receiver has a 4 $\mathrm{mm} \times 6 \mathrm{~mm}$ base and a maximum height of $1.5 \mathrm{~mm}$. Articulatory movements can be tracked within a frequency bandwidth ranging from 0 up to $100 \mathrm{~Hz}$. The receiver electronics includes a high precision linearization circuit which produces signals proportional to the distance between the receivers and the transmitters (Branderud, 1985). In what follows, we describe an experiment designed to estimate the accuracy of the Movetrack under 


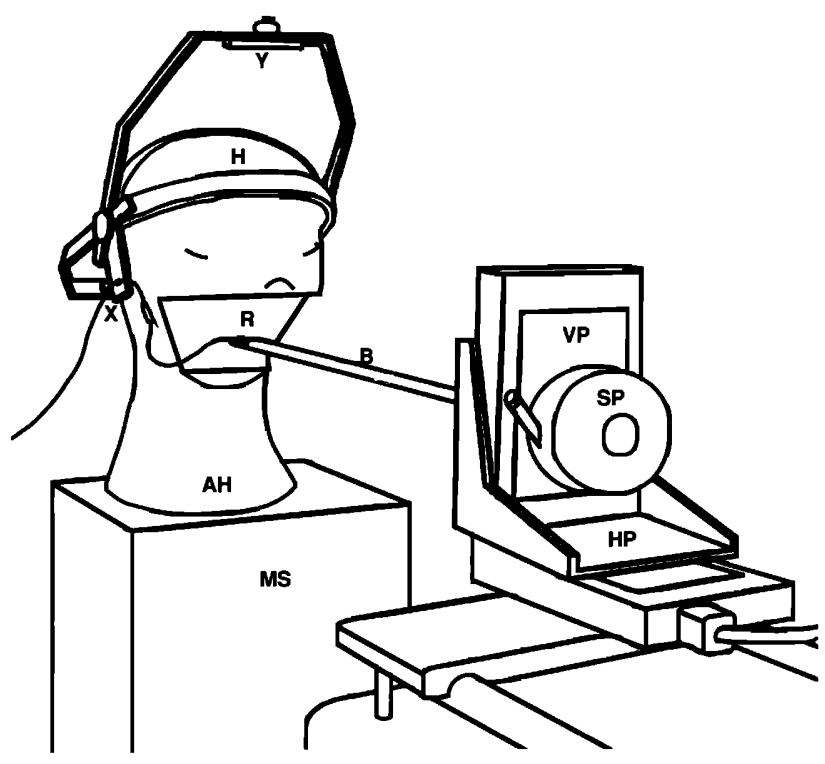

FIG. 1. A schematic diagram of the Movetrack headset and of the calibration bench. $\mathbf{H}$ stands for headset, $\mathrm{X}$ for transmitter $\mathrm{X}$, $\mathrm{Y}$ for transmitter Y, AH for artificial head, MS for movable support, VP for vertical plate, HP for horizontal plate, SP for swivel plate, B for polyacetal bar, $\mathbf{R}$ for receiver.

several conditions of rotational misalignment of a receiver with respect to the transmitters.

\section{METHOD}

The Movetrack system was tested by moving a receiver with a high precision mechanical device in the midsagittal plane. The various components of the device are marketed by Micro-Contrôle (Evry, France). It consists of a cylindrically shaped polyacetal bar (diameter: $1 \mathrm{~cm}$, length: $53 \mathrm{~cm}$ ) fastened to a vertical plate mounted on a track-driven horizontal plate. Each plate is driven by a motor so that the bar can be moved up and down and forward and backward, with a precision of $1 \mu \mathrm{m}$. The maximum range of variation is $5 \mathrm{~cm}$ in the horizontal and vertical directions. The movements of the bar are controlled by a microcomputer. The bar can also be turned manually around its longitudinal axis by means of a movable plate equipped with a vernier $\left(1 / 60^{\circ}\right.$ graduations).

Figure 1 illustrates the way in which the assembly was set up. The Movetrack headset was fastened securely to a polystyrene "head" placed a few centimeters away from the polyacetal bar. A receiver was attached to the end of this bar, whose extremity was cut to form a ledge. The lower front quarter of the head was removed so that the receiver could be moved around in the space approximately defined by the limits of the anterior cavity of the vocal tract. The two transmitter frames were oriented in such a way that the origin of the measurement coordinate system in the midsagittal plane was located approximately in the center of the scanning field. The midsagittal plane was defined as the vertical plane passing in the middle of both transmitters $\mathrm{X}$ and $\mathrm{Y}$. The origin was defined as the point of intersection between the vertical $y$ axis (passing through transmitter $\mathrm{Y}$ ) and the horizontal $x$ axis (passing through transmitter $X$ ) in that plane. In what follows, we will also use the terms "right" and "left" to refer to horizontal displacements of any piece of the assembly perpendicular to the bar, while the terms "forward" and "backward" will designate horizontal displacements parallel to the bar towards the polystyrene head or in the other direction.

The Movetrack receiver module was connected to a 12-bit A/D converter (Analog Connection Jr., Strawberry Tree Inc., Cal.; sampling rate: $1000 \mathrm{~Hz}$ ) linked to a microcomputer. Two output signals were produced by the receiver module. The first, $V_{x}$, was proportional to the distance $d_{x}$ between the receiver and transmitter $X$ : $V_{x}=k_{x} d_{x}+b_{x}$. The second signal, $V_{y}$, was proportional to the distance $d_{y}$ between the receiver and transmitter Y: $V_{y}=k_{y} d_{y}+b_{y}$. The gains, $k_{x}$ and $k_{y}$, and the offsets, $b_{x}$ and $b_{y}$, were adjusted on the receiver module so as to make the best use of the converter range $(-5 \mathrm{~V} /+5 \mathrm{~V})$.

The most important point in setting up this assembly concerns the way in which the transmitters and the receiver were positioned with respect to each other. The procedure we used to minimize off-midline placement and rotational misalignment of the receiver can be described as follows. At first, the receiver was carefully attached to the polyacetal bar so as to be oriented perpendicular to the axis of the bar with its midpoint intersecting that axis. Special markers had been engraved beforehand on the bar to facilitate this operation. A magnifying glass was used to verify that the receiver was correctly placed. The following stage consisted in aligning the bar axis with the midsagittal plane. For this it was necessary to adjust the position of the artificial head itself, since the calibration bench used in the present study did not allow lateral movements of the bar. Inversely, the artificial head had been placed on a movable support which could be shifted laterally and rotated. Thus this support was slightly moved on either side of the bar until the values of $V_{x}$ and $V_{y}$ displayed on the digital voltmeter of the Movetrack receiver module were minimal. The support was then slowly rotated in one direction and in the opposite one, so as to orient the artificial head in such a way that $V_{x}$ and $V_{y}$ had the smallest possible values, all other things being equal. With the head in that position, the distance from the receiver to the midsagittal plane was considered as being close to zero. Finally the transmitters and the receiver had to be properly oriented with respect to each other. One important characteristic of Movetrack must be mentioned in this connection: The transmitters are mounted on their frames in a way that allows the user to swivel them, making it possible for them not to be parallel to one another. This feature enables us to orient the axis of each transmitter to be virtually parallel to a transducer (of course, this can only be done for one transducer; to the extent that multiple transducers are not parallel to one another, all but the one will not be parallel to the transmitters). First the receiver was placed in a roughly horizontal position by turning the polyacetal bar around its longitudinal axis. Then each transmitter was swiveled until the distance from the receiver to that transmitter appeared to be minimal on the Movetrack voltmeter. Finally, the 
receiver was again rotated to minimize further the voltmeter values, as far as this was possible. Once these minimal values were attained, we considered that the tilt angle between the receiver and the transmitters was equal to $0^{\circ}$.

The test was run in five 4-step sessions. The first step in each session was to determine the exact location of the origin $\boldsymbol{O}$ of the measurement coordinate system in the midsagittal plane. This origin must be known in order to calculate the coordinates of the receiver from the measured signals. First, the receiver was moved horizontally in 1-mm steps across an interval of $3 \mathrm{~cm}$. Variations in the $V_{y}$ signal between the bounds of this interval were modeled as a parabolic curve using a polynomial approximation. The location of the origin on the $x$ axis was taken to be the extremum of the curve. The location of the origin on the $y$ axis was determined using the same method, by moving the receiver vertically and noting the value of $V_{x}$ at each point.

The purpose of the second step was to determine the exact value of the gains and offsets. Gain $k_{x}$ was defined as the mean value of the difference $\left(V_{x+1}-V_{x}\right)$, calculated by moving the receiver over a $3-\mathrm{cm}$ interval along the $x$ axis in 1-mm steps. Gain $k_{y}$ was calculated in exactly the same way. The offsets were read directly off the digital voltmeter on the Movetrack calibration module.

The next step consisted of "scanning" a 4-cm square surface in 5-mm steps along the $x$ and $y$ axes. The receiver was thus placed at 81 points forming a square grid. $V_{x}$ and $V_{y}$ were read ten times at each point, and the respective medians of these two series of ten measures were stored in a file. The procedure was repeated five times.

The last step in each session was to calculate the $x$ and $y$ coordinates of the receiver for each $\left(V_{x}, V_{y}\right)$ pair of measures. The location of the receiver was taken to be the intersection point between circle $C_{x}$ of radius $d_{x}$ centered on transmitter $\mathrm{X}$, and circle $C_{y}$ of radius $d_{y}$ centered on transmitter Y. $C_{x}$ and $C_{y}$ intersect at two different points, but it is easy to determine which of the two is in the displacement plane of the receiver.

The difference between one session and the following one lies in the tilt angle of the receiver. This angle was changed before doing the scanning to $10^{\circ}$ in the second session, $15^{\circ}$ in the third, $20^{\circ}$ in the fourth, and $25^{\circ}$ in the fifth. It was always equal to $0^{\circ}$ during the localization of the origin and the calculation of the gains and offsets.

\section{RESULTS}

The results indicated that the absolute precision of the measures is very satisfactory when the tilt is $0^{\circ}$ (session 1 ). The mean distance between the calculated location of the receiver and its real location was $0.35 \mathrm{~mm}$, and there was very little variation in this distance across points or repetitions $(\sigma=0.05)$. In fact, the error proved to be due essentially to a constant $x$ and $y$ offset. By "centering" the reference grid in such a way that it coincided at its midpoint with the center of gravity of the data (having $x$ and $y$ grand average values as coordinates) in the midsagittal plane, it was possible to reduce this error to less than $\mathbf{0 . 2}$ $\mathrm{mm}$. This demonstrates that the relative precision of the system is excellent, provided the tilt is zero degrees.
TABLE I. Average distances (in $\mathrm{mm}$ ) between the measured and real positions of the receiver coil for five different degrees of tilt, with the associated standard deviations. Each average distance was calculated by scanning five times a $4 \times 4-\mathrm{cm}$ grid composed of 81 equidistant points in the midsagittal plane $(n=81 \times 5)$.

\begin{tabular}{ccccc}
\hline \hline Session & Tilt (deg) & $n$ & Dist. (mm) & s.d. \\
\hline 1 & 0 & 405 & 0.35 & 0.05 \\
2 & 10 & 405 & 1.29 & 0.16 \\
3 & 15 & 405 & 2.42 & 0.22 \\
4 & 20 & 405 & 5.36 & 0.29 \\
5 & 25 & 405 & 9.34 & 0.36 \\
\hline \hline
\end{tabular}

Table I also shows that the difference between the measured and real position of the receiver increases rather substantially as a function of the angle of tilt $\left(10^{\circ}, 15^{\circ}, 20^{\circ}\right.$, $25^{\circ}$ ). In session 2 and the following ones, the receiver appeared at each point to be farther than it actually was from the transmitters. This result is consistent with the theoretical relationship between the voltage of the signal induced in that receiver for each transmitter and the tilt angle (see above).

\section{CONCLUSION}

This study showed that the measurements of position made by the Movetrack system are highly accurate when the receiver is placed in the midsagittal plane and is parallel to the two transmitters. When such conditions are met, the measurement error appears to be on average lower than $0.4 \mathrm{~mm}$. Moreover, it is possible that the accuracy of the system was slightly underestimated in our test, since the method we used to position the receiver and the transmitters with respect to each other probably introduced some imprecision into our measurements. Thus the $x$ and $y$ offset observed could possibly be explained by the fact that the tilt angle was not strictly equal to $0^{\circ}$ in the first session, or that the receiver was slightly outside the midline plane. Furthermore, it has to be noted that our measurements were made in the peripheral area of the articulatory space (near the mouth opening). The error can be assumed to be even smaller at locations closer to the transmitters. The results may also be further improved by using a more accurate voltmeter for measuring the offsets.

However, Movetrack has two major drawbacks. First of all, the exact location of the origin of the measurement coordinate system in the midsagittal plane must be determined at the beginning of each experiment. This origin does not maintain a fixed position due to the fact that the two frames carrying the transmitters may rotate around their fixation points on the headset (their orientation is adjusted by screws near the temples). One of the goals of the present work was to propose a method suitable for locating the origin before the headset is placed on the subject's head. This problem is obviously a delicate one and seems difficult to solve without the aid of a rather complex calibration bench like the one described above.

Second, and more importantly, the device does not offer the possibility of compensating for rotational misalignment of the receivers. This might be of secondary im- 
portance for receivers that are attached to anatomically rigid structures like the lower jaw for example, since, in that case, tilt can mainly be caused by a bad placement of the receiver on the articulator, and should remain constant during experimentation, thus resulting in simple $x$ and $y$ offset. But the tongue and lips are soft bodies whose deformation during the production of speech may produce a nonconstant, time-varying receiver tilt. In this second case, the difference between the measured and the real position of the receiver will vary itself as function of the degree of tilt. Since the Movetrack does not perform any tilt detection, the accurate measurements (no tilt) will be inextricably mixed with the unreliable ones (large amount of tilt). In other words, it will be difficult to know whether a variation of the receiver output is due to a real displacement of that receiver in the midsagittal plane, or merely to a variation in the degree of tilt.

The result is that the usefulness of the Movetrack for collecting quantitative data on the movements of tongue and lips depends to a large extent on how much tilt can be produced by such nonrigid structures. Unfortunately, we do not yet have very precise data on that point. More extensive studies are needed, which would consist for example in combining EMA with cineradiography, so as to compare the actual position of a receiver (visible on $\mathrm{x}$ ray) to its calculated position. Ultrasound imaging would also be a suitable method for estimating the maximal angle of tilt of a receiver attached to the tongue, since it can provide cross-sectional views of the tongue in a plane perpendicular to the midline plane. The EMA devices which are designed to detect and to compensate for tilt should themselves allow us to obtain more and more reliable data on rotational movements of the receivers, as empirical studies using such devices become more frequent (Perkell et al., 1992; Hoole, 1993). At present however, it would be premature to claim that deformations of the tongue or lips do not sufficiently modify the orientation of an electromagnetic transducer to alter the accuracy of measurements in a significant way.

In conclusion, our results showed that the Movetrack allows us to determine the position of a receiver in the midline plane with high accuracy, as long as this receiver is not tilted. Thus the device should be suitable for tracking movements of rigid anatomical structures like the lower jaw. However, applications to the quantitative analysis of tongue and lips movements should be deferred until it can be proven that such soft structures do not cause significant rotational misalignments of the receivers.

\section{ACKNOWLEDGMENTS}

This study is part of the European ESPRIT/ACCOR research project. The tests were run in the Marseille L.A.S. (Space Astronomy Laboratory), which kindly gave us access to its calibration bench. We are grateful to NguyenTrong Thanh, head of the L.A.S. Electronics Division for his valuable contribution. We also thank P. Branderud, $U$. Frauenfelder, P. Hoole, W. Jones, K. Nicolaidis, J. Perkell, B. Teston, and D. Ward for helpful comments.

Branderud, P. (1985). "Movetrack—a movement tracking system," in Proceedings of the French-Swedish Symposium on Speech, GALF, Grenoble, France, 22-24 April 1985, pp. 113-122.

Hixon, T. J. (1971). "An electromagnetic method for transducing jaw movements during speech," J. Acoust. Soc. Am. 49, 603-606.

Hoole, P. "Electromagnetometric techniques," in Instrumental Studies of Coarticulation, edited by W. J. Hardcastle (Cambridge U.P., Cambridge, England) (in press).

Perkell, J. S., and Cohen, M. H. (1986). “An alternating magnetic field system for tracking multiple speech articulatory movements in the midsagittal plane," Tech. Rep. No. 512, MIT, Cambridge, MA.

Perkell, J. S., Cohen, M. H., Svirsky, M. A., Matthies, M. L., Garabieta, I., and Jackson, M. T. T. (1992). "Electromagnetic midsagittal articulometer (EMMA) systems for transducing speech articulatory movements," J. Acoust. Soc. Am. 92, 3087-3096.

Schönle, P., Gräbe, K., Wenig, P., Höhne, J., Schrader, J., and Conrad, B. (1987). "Electromagnetic articulography: use of alternating magnetic fields for tracking movements of multiple points inside and outside the vocal tract," Brain Lang. 31, 26-35.

Stone, M. (1991). "Towards a model of three-dimensional tongue movement," J. Phon. 19, 309-320.

Tuller, B., Shao, S., and Kelso, J. A. S. (1990). "An evaluation of an alternating magnetic field device for monitoring tongue movements," J. Acoust. Soc. Am. 88, 674-679.

van der Giet, G. (1977). "Computer-controlled method for measuring articulatory activities,” J. Acoust. Soc. Am. 61, 1072-1076. 